\title{
Comparison of Brans Promethee multicriteria decision method and Promethee modified by authors for the optimization of an erosion control integral plan in Chaco area in Salta province (Argentine)
}

\author{
J. B. Grau (1), J. M. Antón (1), F. Colombo (2), L. de los Ríos (2), J. M. Cisneros (3), A. Tarquis (1) \\ (1) E. T. S. I. Agrónomos- Universidad Politécnica de Madrid (Spain) (2) Escuela de Negocios- Universidad Católica de \\ Salta (Argentina) (3) Facultad de Agronomía y Veterinaria-Universidad Nacional de Río Cuarto (Argentina) \\ E-mail: j.grau@upm.es
}

\begin{abstract}
Chaco area is situated in the Province of Salta at North West of Argentine. The desertification is a big problem. In order to mitigate the problem it is necessary to take into account not only pedologic criteria but the economical, environmental, cultural and sociological criteria. Six sub zones have been established following previous studies. Eight criteria and six alternatives have been introduced in the model. Following the results of the study carried out by a collaborative project between UPM and UCS financed by AECID (1) were established several initial matrix. Brans Promethee Multicriteria Decision Method (MCDM) was applied and the authors modified that method introducing weights like in Electre Method.
\end{abstract}

\section{INTRODUCTION}

The Salta Province has $155.000 \mathrm{~km}^{2}$ and 1 million population, it is at NW of Argentine (NOA) having latitudes around $25^{\circ} \mathrm{S}$, it has rain from 400 to $800 \mathrm{~mm} /$ year. It has a low density of population in small cities and Indian places "puestos" or "colonias". It has low standards for roads and it has an environment that is "deteriorating progressively".

Water is the most critical factor, as much for human and animal consumption, as for the production system in general and for the flooding and lack of appropriated infrastructures.

Besides the water, other factors have an important influence in the erosion and progressive desertification of this region and environment degradation. Historically the human exploitation of natural forest to use in the railway and other activities produced an environment degradation process. Later on the autochthonous population followed the irrational wood extraction an over pasture as "modus vivendi" contributing to make the situation worse. Actually the farms and big single-crop exploitations in some locations do not give solution to the desertification problem. Only one integral plan considering all factors involved and the differences among sub zones will be an initial point to change the direction of the desertification process.

\section{A. Criteria, alternatives and Sub zones}

The following eight criteria were defined:

Water erosion (WE): The water erosion is important. The relative water erosion indexes figures in the decisional matrix.

Eolian erosion (EE): Winds erode, transport and deposit materials and are effective agents in several areas of this region.

Implementation Facility (IF): They have been established taking into account actors' opinions.

Water Resources (WR): By each alternative have been considered and the relative results have been taken into this criterion.

Economical benefits EB): The relative economical benefits using each alternative in a period of 25 years have been obtained as shown in the matrix with figures from 1 to 10 .

Hand power (HP): We have considered that would be satisfactory to give employment to the majority of it population. For that, we have considered this criterion as of "more is better" kind.

Environmental Impacts (EI): They have been considered in each sub zone the environmental impacts according with the alternative adopted.

Social Acceptance (SA): The figures included in this criterion have been obtained from the results of different forums and meeting with institutions, organizations and native people.

Five alternatives have been retained:

A) Autochthonous forest: mainly "Quebracho Blanco" and "Quebracho Colorado" forest species.

B) High value forest: mainly teak, ebony, walnut tree, cherry tree, lignum vitae, eucalyptus, etc

C) Traditional farms with extensive agriculture and livestock mixed with autochthonous forest modified and several foraging plants.

D) Erosion control Crop with agriculture use.

E) Erosion control crop with industrial use (biomass).

Following the experience and the local acknowledge, the area has been divided in 6 sub zones: Las Lajitas, La Estrella, Pichanal, Martin Hickmann, Rivadavia banda sur and Joaquín V. Gonzalez.

\section{METHODOLOGY}

We have used the Preference Ranking Organization Method (The PROMETHEE Method for Multiple Criteria DecisionMaking) by Ref. $[4,13,14]$. This is an outranking method, as 
ELECTRE due to Roy $[11,12]$ or A.H.P. due to Saaty [15, 16, 17]. Following Ref. [4, 13, 14] two possibilities are offered, PROMETHEE I provides a partial preorder and PROMETHEE II a total preorder on the set of possible alternatives. Different types of criteria have been adopted.

Type I and Type III with different threshold (m). Type I is the usual Criterion. With this criterion if $f(a)=f(b)$ this is indifference between $a$ and $b$. If this is not the case the decision-maker has a strict preference for the action having greatest value. Type III is the Criterion with Linear Preference. Such an extension of the notion of criterion allows the decision-maker to prefer progressively $a$ to $b$ for progressively larger deviations between $f(a)$ and $f(b)$. The preference increases linearly until deviation equals $\mathrm{m}$, after this value the preference is strict. For $m$ the values 2, 4 and 6 have been taken.

The authors have modified the PROMETHEE method using the weights of the criteria following the ELECTRE I Method $[6,1,2,3,7,8,9,10]$. In the case I have been adopted the same weights for all sub zones and in the case II different weights

Besides, some modifications have been considered in the data of the initial matrixes.

Finally, MathCad has been used to program the calculus.

We show below, like example, the application to sub zone "La Estrella".

EROSION AND DESERTIFICATION INTEGRAL CONTROL PLAN USING PROMETHEE$$
\text { SUB ZONE LA ESTRELLA }
$$$$
\text { (1) }
$$$$
\text { ORIGIN: }=1
$$
CRITERION: 1.-water erosion index 2.- eolian erosion index, 3.- Imp
facility 4.-Water Resources, 5.- Economical Benefits, 6.- Hand power,
7.-Environmental Impacts, 8.- Social Acceptance

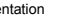

$\frac{\text { Alternatives: }}{\mathrm{i}=1 \ldots 5}$

A- functions of criterion-parameter and type elected for each criterion $j$ : following Ref. [4] $j=6$ type III, $m=6, j=7$ type III, $m=6 j=8$ type III, $m=2$

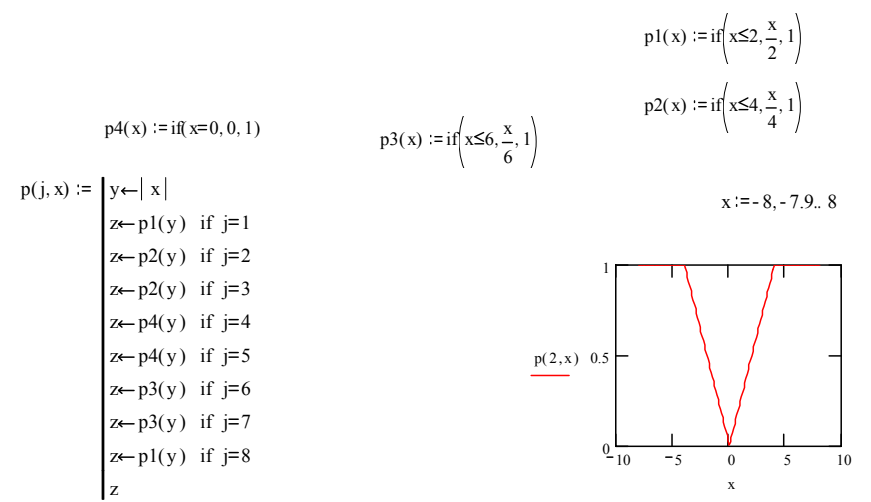

más es mejor Isubj $=1$ más es peor Isubj = -1

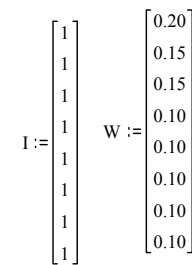

with $x>=0$, if not with $|x|$
Índixes $q(i, i i)$ of preferences $(\pi(i, i i)$ Brans\&Vincke),

giving outranking graphs according with values

$q(i, i i):=\frac{\sum_{j=1}^{8} P(i, i i, j)}{8}$

$\mathrm{i}:=1 . .5 \quad$ ii $:=1 . .5 \quad \mathrm{qq}_{\mathrm{i}, \mathrm{ii}}:=\mathrm{q}(\mathrm{i}, \mathrm{ii})$

$\mathrm{qq}=\left[\begin{array}{ccccc}0 & 0.229 & 0.448 & 0.438 & 0.458 \\ 0.25 & 0 & 0.281 & 0.333 & 0.354 \\ 0.5 & 0.281 & 0 & 0.406 & 0.156 \\ 0.208 & 0.094 & 0.042 & 0 & 0.021 \\ 0.458 & 0.469 & 0.208 & 0.5 & 0\end{array}\right]$

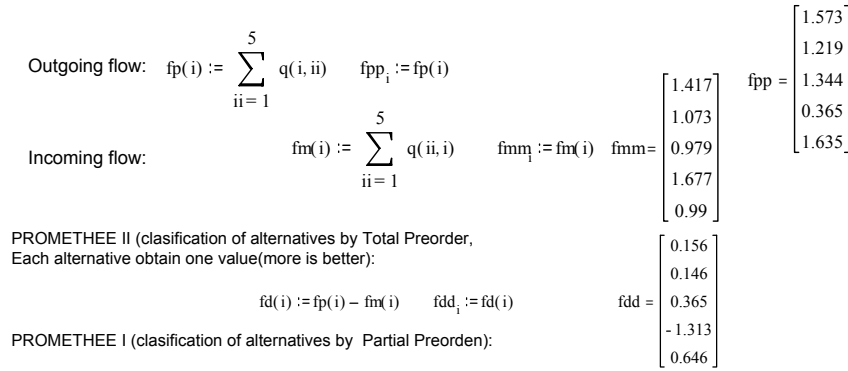

$\operatorname{pr}(\mathrm{i}, \mathrm{ii}):=\mid \mathrm{z} \leftarrow-1$

$\mathrm{z \leftarrow 0}$ if $(\mathrm{fp}(\mathrm{i})=\mathrm{fp}(\mathrm{ii}) \cdot(\mathrm{fm}(\mathrm{i})=\mathrm{fm}(\mathrm{ii})$

$z \leftarrow 1$ if $((f p(i)>f p(i i)) \cdot(f m(i)<f m(i i)))+((f p(i)>f p(i i)) \cdot(f m(i)=f m(i i)))+((f p(i)=f p(i i)) \cdot(f m(i)<f m(i i)))$

$\operatorname{prr}_{i, i \mathrm{i}}:=\operatorname{pr}(\mathrm{i}, \mathrm{ii})$

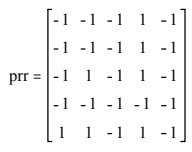

Alternative $E$ is preferred (E C A B D)

Results following the method modified by Ref [6] in order to weigh comparativment the criteria

with similar weights to ELECTRE-l:

q Preference Index ( $\pi$ Ref. [4]), gives outranking graph by values

$\mathrm{i}:=1 . .5 \quad$ ii $:=1 . .5$

$\mathrm{qq}_{\mathrm{i}, \mathrm{ii}}:=\mathrm{q}(\mathrm{i}, \mathrm{ii}) \cdot 5$

$q(i, i i):=\sum_{j=1}^{8} P(i, i i, j) \cdot w_{j}$

$q \mathrm{q}=\left[\begin{array}{ccccc}0 & 0.917 & 2.479 & 2.5 & 2.583 \\ 1.25 & 0 & 1.813 & 2.083 & 2.167 \\ 2.25 & 1.188 & 0 & 1.938 & 0.688 \\ 1.083 & 0.438 & 0.167 & 0 & 0.083 \\ 2.083 & 2.063 & 0.958 & 2.375 & 0\end{array}\right]$

$\begin{array}{lll}\text { Outgoing flow: } & \mathrm{fp}(\mathrm{i}):=\sum_{\mathrm{ii}=1}^{5} \mathrm{q}(\mathrm{i}, \mathrm{ii}) & \mathrm{fpp}_{\mathrm{i}}:=\mathrm{fp}(\mathrm{i}) \quad \mathrm{fpp}=\left[\begin{array}{l}1.696 \\ 1.463 \\ 1.213 \\ 0.354 \\ 1.496\end{array}\right] \quad \mathrm{fmm}=\left[\begin{array}{l}1.333 \\ 0.921 \\ 1.083 \\ 1.779 \\ 1.104\end{array}\right]\end{array}$

PROMETHEE II (clasification of alternatives by Total Preorder)

$$
\mathrm{fd}(\mathrm{i}):=\mathrm{fp}(\mathrm{i})-\mathrm{fm}(\mathrm{i}) \quad \mathrm{fdd}_{\mathrm{i}}:=\mathrm{fd}(\mathrm{i})
$$$$
\text { fdd }=\left[\begin{array}{c}
0.363 \\
0.542 \\
0.129 \\
-1.425 \\
0.392
\end{array}\right]
$$

PROMETHEE I (clasification of alternatives by Partial Preorden):

$\operatorname{pr}(\mathrm{i}, \mathrm{ii}):=\mid \begin{aligned} & \mathrm{z}--1 \\ & \mathrm{z}-0 \text { if }(\mathrm{fp}(\mathrm{i})=\mathrm{fp}(\mathrm{ii})) \cdot(\mathrm{fm}(\mathrm{i})=\mathrm{fm}(\mathrm{ii})) \\ & \mathrm{z}-1 \text { if }((\mathrm{fp}(\mathrm{i})>\mathrm{fp}(\mathrm{iii}) \cdot(\mathrm{fm}(\mathrm{i})<\mathrm{fm}(\mathrm{iii}))+\end{aligned}$

$\mathrm{z}_{\mathrm{Z}-1}$ if $((\mathrm{fp}(\mathrm{i})>\mathrm{fp}(\mathrm{ii})) \cdot(\mathrm{fm}(\mathrm{i})<\mathrm{fm}(\mathrm{ii})))+((\mathrm{fp}(\mathrm{i})>\mathrm{fp}(\mathrm{ii})) \cdot(\mathrm{fm}(\mathrm{i})=\mathrm{fm}(\mathrm{ii})))+((\mathrm{fp}(\mathrm{i})=\mathrm{fp}(\mathrm{ii})) \cdot(\mathrm{fm}(\mathrm{i})<\mathrm{fm}(\mathrm{ii})))$

where, $\operatorname{pr}(\mathrm{i}, \mathrm{ii})=1$ tell us that alternative $\mathrm{i}$ is preference (outranks) to alternative $\mathrm{j}, \operatorname{pr}(\mathrm{i}, \mathrm{ii})=0$ is indifference, $y$ pr(i,ii) $=-1$ are incomparable, that may be obtained by pr(ii,i)

$\operatorname{prr}_{i, \text { ii }}:=\operatorname{pr}(\mathrm{i}, \mathrm{ii}) \quad \operatorname{prr}=\left[\begin{array}{ccccc}-1 & -1 & -1 & 1 & -1 \\ -1 & -1 & 1 & 1 & -1 \\ -1 & -1 & -1 & 1 & -1 \\ -1 & -1 & -1 & -1 & -1 \\ -1 & -1 & -1 & 1 & -1\end{array}\right]$ 


\section{RESULTS}

\section{Sub zone Martin Hickman}

\subsection{ALTERNATIVE1:}

Table I shows the values to Martin Hickman sub-zone. It has been included the type of pseudo-criteria used and the threshold (m) for the type III [4].

TABLE I

DeCisional Matrix For MARTIN HICKMAN, ALTERNATIVE 1

\begin{tabular}{|l|c|c|c|c|c|c|c|c|}
\hline Alternative & WE & EE & IF & WR & EB & HP & EI & SA \\
\hline A & 9 & 8 & 5 & 8 & 7 & 2 & 8 & 2 \\
\hline B & 7 & 5 & 7 & 5 & 6 & 8 & 6 & 5 \\
\hline C & 4 & 2 & 8 & 4 & 8 & 8 & 1 & 9 \\
\hline D & 3 & 3 & 6 & 4 & 6 & 7 & 5 & 6 \\
\hline E & 3 & 3 & 2 & 6 & 8 & 6 & 5 & 8 \\
\hline Weight & 0,2 & 0,2 & 0,05 & 0,1 & 0,1 & 0,1 & 0,15 & 0,1 \\
\hline Type of criterion & III & III & III & I & I & III & III & III \\
\hline Thresholds & 2 & 4 & 4 & & & 2 & 2 & 4 \\
\hline
\end{tabular}

Two procedures have been applied in order to obtain alternative preorder:

A: Initial method of Ref. [4].

B: Method modified by Ref. [6].

1.1.A. Results by Ref. [4] method. The preorder is shown in Fig. 1.

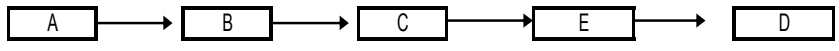

Fig. 1 Graph sub zone Martin Hickman, alternative 1.1A, Promethee II.

1.1. B Results following Ref. [9] method are shown in Fig. 2.

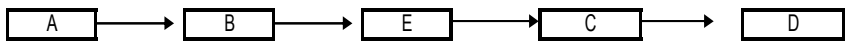

Fig. 2: Graphs sub zone Martin Hickman 1.1B Promethee II, modified.

1.2 ALTERNATIVE 2: Other value of criteria, same weight, pseudocriteria and thresholds (Table II andy Fig. 3 and 4).

TABLE II

DeCisional MATRIX For MARTin HickMAn, ALternative 2.

\begin{tabular}{|l|c|c|c|c|c|c|c|c|}
\hline Alternative & WE & EE & IF & WR & EB & HP & EI & SA \\
\hline A & 9 & 8 & 5 & 8 & 7 & 7 & 8 & 2 \\
\hline B & 7 & 5 & 7 & 5 & 8 & 8 & 6 & 5 \\
\hline C & 7 & 5 & 8 & 4 & 8 & 8 & 6 & 9 \\
\hline D & 3 & 3 & 6 & 4 & 6 & 7 & 5 & 6 \\
\hline E & 3 & 3 & 8 & 6 & 8 & 6 & 5 & 8 \\
\hline Weight & 0,2 & 0,2 & 0,05 & 0,1 & 0,1 & 0,1 & 0,15 & 0,1 \\
\hline Type of criterion & III & III & III & I & I & III & III & III \\
\hline Thresholds & 2 & 4 & 4 & & & 2 & 2 & 4 \\
\hline
\end{tabular}

1.2. A. Results by method of Ref. [4] method. The preorder is shown in Fig. 3.

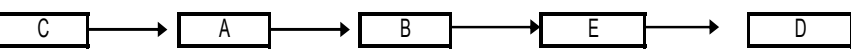

Fig. 3: Graphs sub zone Martin Hickman, alternative 1.2.A . Promethee II

1.2. B. Results following Ref [9] method are shown in Fig. 4.

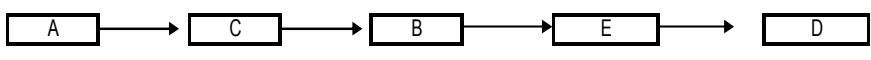

Fig. 4: Graphs sub zone Martin Hickman, alternative 1.2.B. Promethee II, modified.

Changing the weights $(0,2-0,15-0,15-0,10-0,10-0,10$
$-0,10-0,10)$, the results are:

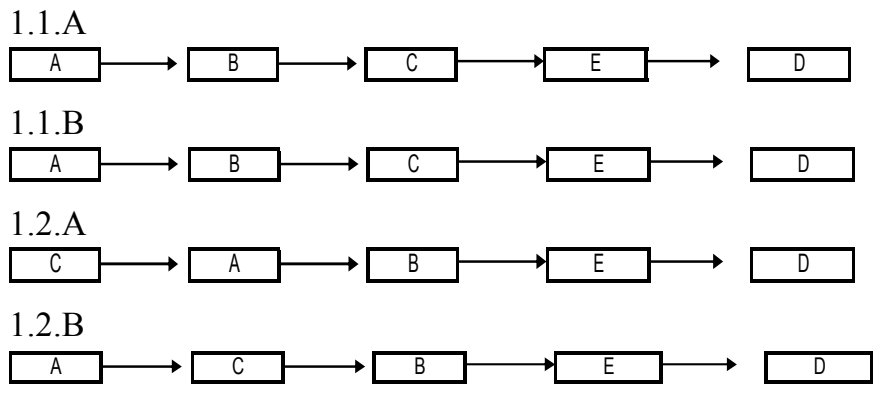

\section{Sub zone LA ESTRELLA}

2.1. ALTERNATIVE 1: Decisional matrix is shown in Table III.

TABLE III

DeCisional Matrix For LA ESTRELla, ALTERNATIVE 1.

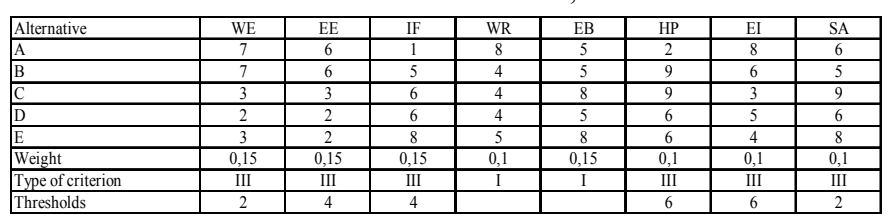

2.1A. Results by Ref [4] method. The preorder is shown in Fig. 5.

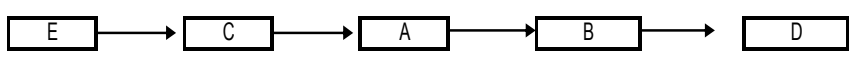

Fig. 5: Graph sub zone La Estrella alternative 2.1A Promethee II

2.1B. Results following Ref [6] method are shown in Fig. 6.

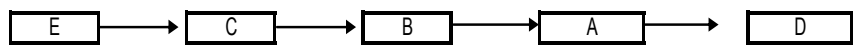

Fig. 6: Graphs sub zone La Estrella alternative 2.1B Promethee II, modified.

2.2. ALTERNATIVE 2: Changing some criteria values and maintaining weights (Table IV). TABLE IV

DECISIONAL MATRIX FOR LA ESTRELla, ALTERNATIVE 2

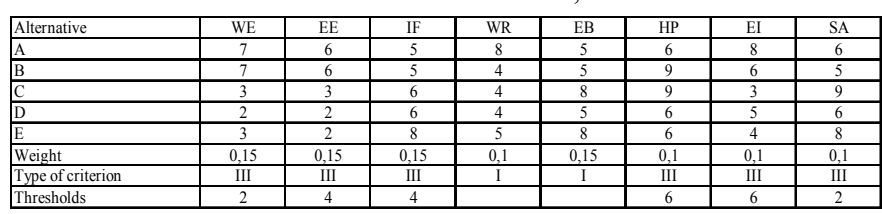

2.2A. Results by Ref. [4] method. The preorder is shown in Fig. 7.

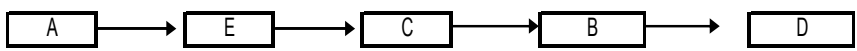

Fig. 7: Graph sub zone La Estrella, alternative 2.2A, Promethee II.

2.2B.Results following Ref [6] method is shown in Fig. 8.

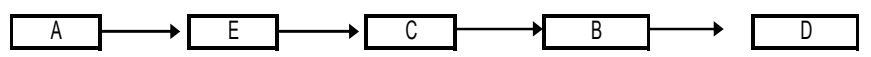

Fig. 8: Graph sub zone La Estrella alternativa 2.2B, Promethee II modified.

Changing weights $(0,20-0,15-0,15-0,10-0,10-0,10-$ $0,10-0,10)$ the results are:

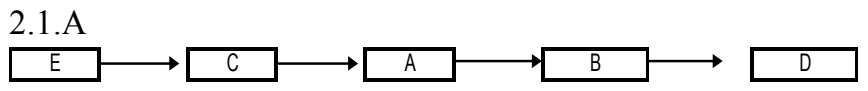


2.1.B

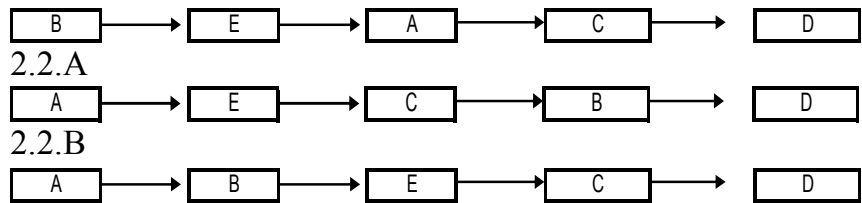

\section{Sub zone RIVADAVIA SUR}

3.1. ALTERNATIVA 1: Initial matrix is shown in Table V.

TABLE V

DECISIONAL MATRIX For RiVADAVIA SUR, ALTERNATIVE 1

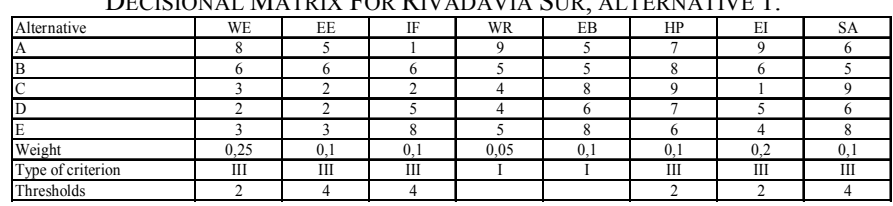

3.1A. Results following Ref [4] method is shown in Fig. 9.

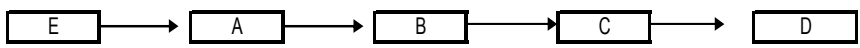

Fig. 9: Graph sub zone Rivadavia Sur, alternative 3.1A, Promethee II

3.1B. Results following Ref. [6] method in Fig. 10.

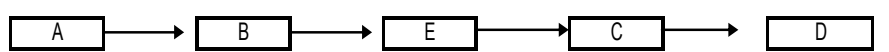

Fig. 10: Graph sub zone Rivadavia Sur, alternative 3.1B, Promethee II, modified.

3.2. ALTERNATIVE 2: Changing some criteria values and maintaining weights (Table VI).

TABLE VI

DECISIONAL MATRIX For RIVADAVIA SUR, ALTERNATIVE 2.

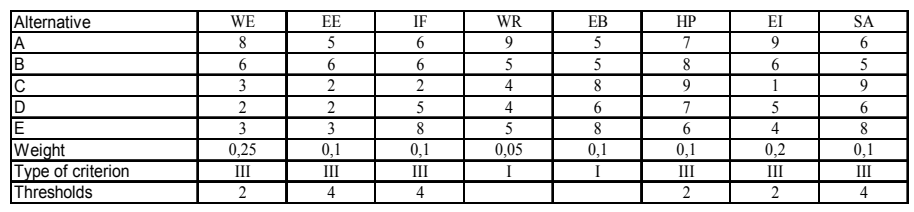

3.2A. Results following Ref. [4] method in shown in Fig. 11.

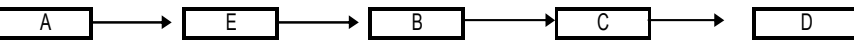

Fig. 11: Graph sub zone Rivadavia Sur, alternative 3.2A, Promethee II.

3.2B. Results following Ref. [6] is in Fig. 12.

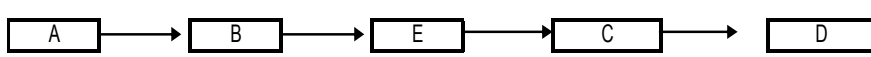

Fig. 12: Graph sub zone Rivadavia Sur, alternative 3.2B, Promethee II, modified.

Changing weights $(0,2-0,15-0,15-0,10-0,10-0,10-$

$0,10-0,10)$ the results are:
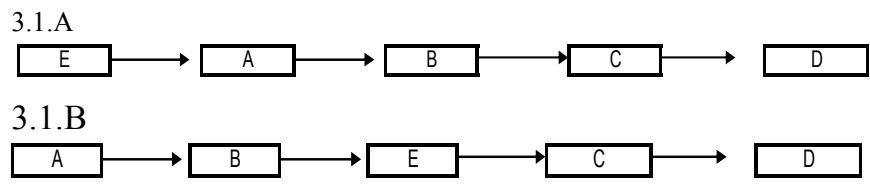

3.2.A

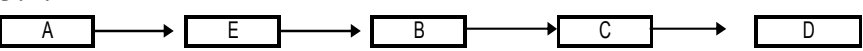

3.2.B

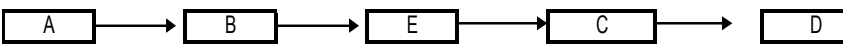

\section{Sub zone PICHANAL}

4.1. ALTERNATIVE 1: Initial matrix is shown in Table VII.

TABLE VII

DeCiSIONAL MATRIX For Pichanal, ALTERNATIVE 1

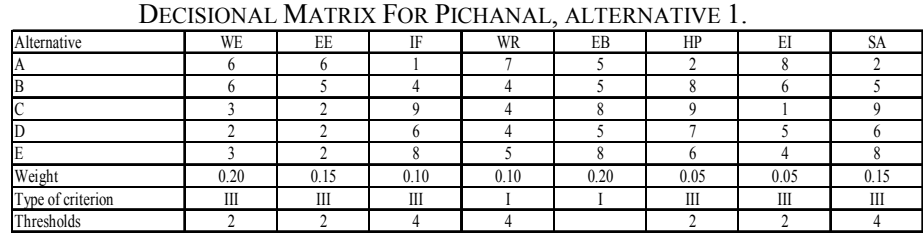

4.1A. Results following Ref. [4] is shown in Fig. 13.

$\underset{\text { Fig. 13: Graph sub zone Pichanal, alternative 4.1A, Promethee II. }}{\longrightarrow}$

4.1B. Results following Ref [6] is shown in Fig. 14.

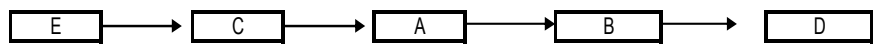

Fig. 14: Graph sub zone Pichanal, alternative 4.1B, Promethee II, modified.

4.2. ALTERNATIVE 2: With other values same weights (Table VIII).

TABLE VIII

Decisional Matrix For Pichanal, ALternative 2.

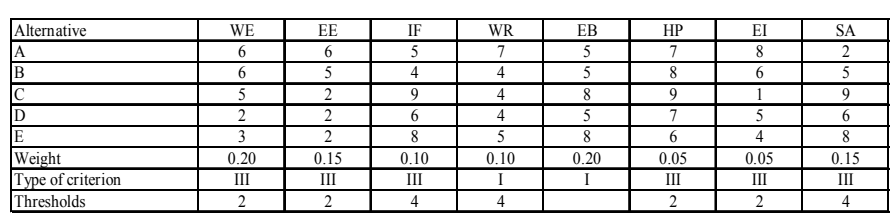

4.2A. Results following Ref [4] is in Fig. 15.

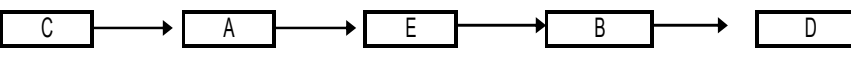

Fig. 15: Graph sub zone Pichanal, alternative 4.2A, Promethee II. 4.2B. Results following Ref [4] is shown in Fig. 16.

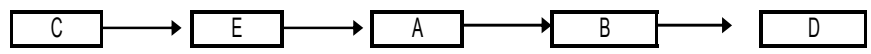

Fig. 16: Graph sub zone Pichanal, alternative 4.2B, Promethee II, modified.

With other weights $(0,2-0,15-0,15-0,10-0,10-0,10-$ $0,10-0,10)$ the results are:

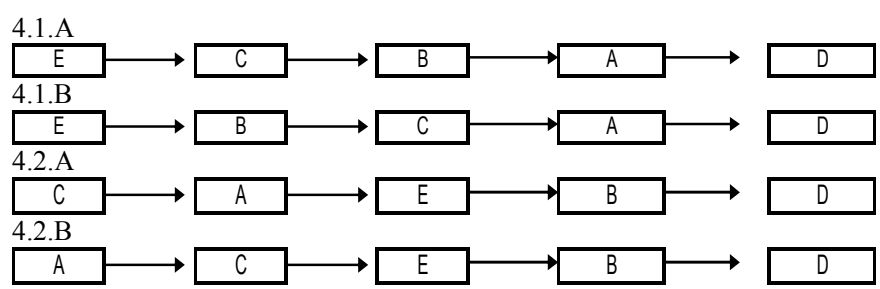

\section{Sub zone JOAQUIN V. GONZALEZ}

5.1. ALTERNATIVE 1: Initial matrix is shown in Table IX. 
TABLE XI

TABLE IX

DECISIONAL MATRIX For JOAQUIN V. GONZÁLEZ, ALTERNATIVE 1.

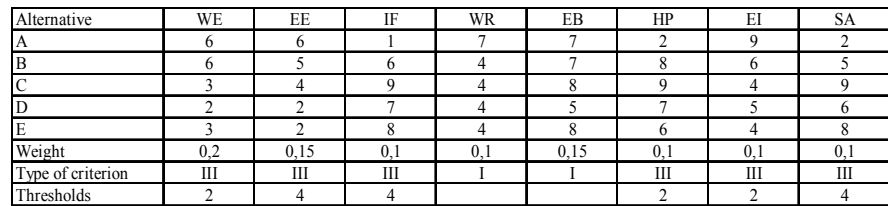

5.1A. Results following Ref. [4] is shown in Fig. 17.

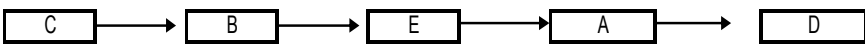

Fig. 17: Graph sub zone Joaquin V. Gonzalez, alternative 5.1A, Promethee

5.1B. Results following Ref. [6] is in Fig. 18.

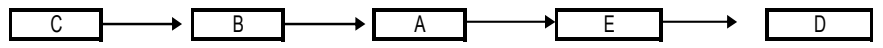

Fig. 18: Graph sub zone Joaquin V. Gonzalez, alternative 5.1B, Promethee II, modified.

5.2. ALTERNATIVE 2: Other values and same weights (Table X).

TABLE X

DECISIONAL MATRIX For JOAQUIN V. GONZÁLEZ, ALTERNATIVE 2.

\begin{tabular}{|l|c|c|c|c|c|c|c|c|}
\hline Alternative & WE & EE & IF & WR & EB & HP & EI & SA \\
\hline A & 6 & 6 & 3 & 7 & 7 & 4 & 9 & 2 \\
\hline B & 6 & 5 & 6 & 4 & 7 & 8 & 6 & 5 \\
\hline C & 5 & 4 & 9 & 4 & 8 & 9 & 4 & 9 \\
\hline D & 2 & 2 & 7 & 4 & 5 & 7 & 5 & 6 \\
\hline E & 3 & 2 & 8 & 4 & 8 & 6 & 4 & 8 \\
\hline Weight & 0,2 & 0,15 & 0,1 & 0,1 & 0,15 & 0,1 & 0,1 & 0,1 \\
\hline Type of criterion & III & III & III & I & I & III & III & III \\
\hline Thresholds & 2 & 4 & 4 & & & 2 & 2 & 4 \\
\hline
\end{tabular}

5.2A. Results following Ref. [4] is shown in Fig. 19.

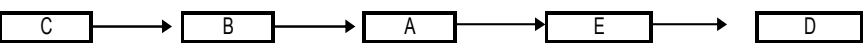

Fig. 19: Graph sub zone Joaquin V. Gonzalez, alternative 5.2A, Promethee II.

5.2B. Results following Ref. [6] is in Fig. 20.

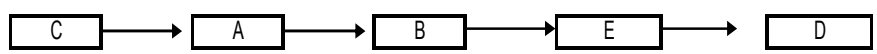

Fig. 20: Graph sub zone Joaquin V. Gonzalez, alternative 5.2B, Promethee II, modified.

With other weights $(0,2-0,15-0,15-0,10-0,10-0,10-$

$0,10-0,10)$ the results are:

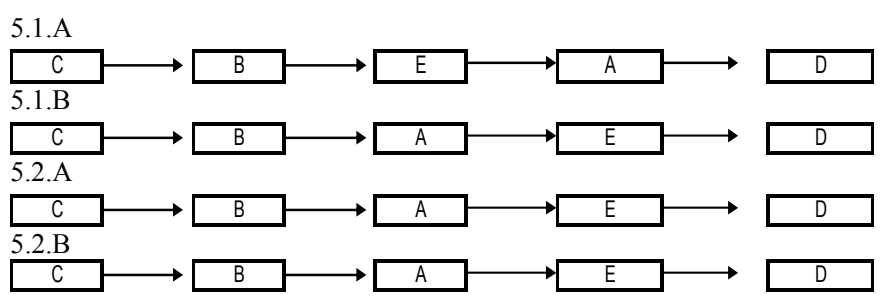

\section{Sub zone LAS LAJITAS:}

6.1. ALTERNATIVE 1: Initial matrix is shown in Table XI.
DECISIONAL MATRIX FOR LAS LAJITAS, ALTERNATIVE 1.

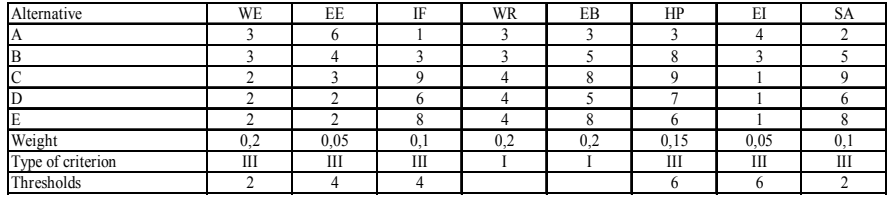

6.1A. Results following Ref. [4] method is in Fig. 21.

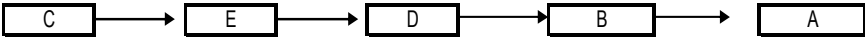

Fig. 21: Graph sub zone Las Lajitas, alternative 6.1A, Promethee II.

6.1B. Results following Ref. [6] method is shown in Fig. 22.

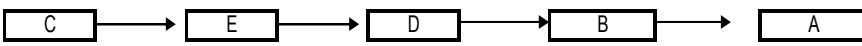

Fig. 22: Graph sub zone Las Lajitas, alternative 6.1B, Promethee II, modified.

6.2. ALTERNATIVE 2: Other values and same weights (Table XII).

TABLE XII

DECISIONAL MATRIX FOR LAS LAJITAS, ALTERNATIVE 2.

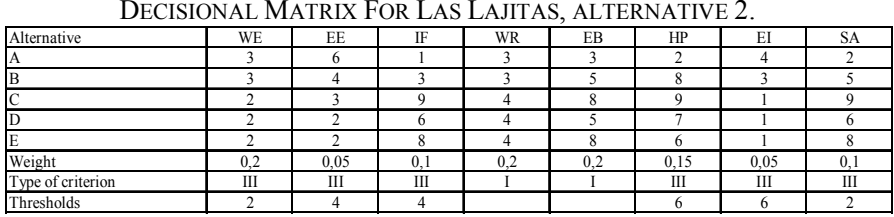

6.2A. Results following Ref. [4] method is shown in Fig. 23.

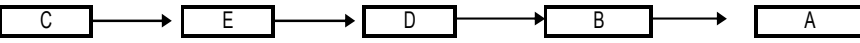

Fig. 23: Graph sub zone Las Lajitas, alternative 6.2A, Promethee II.

6.2B. Results following Ref. [6] method is in Fig. 24.

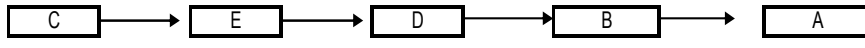

Fig. 24: Graph sub zone Las Lajitas, alternative 6.2B, Promethee II, modified.

Changing weights $(0,2-0,15-0,15-0,10-0,10-0,10-$ $0,10-0,10)$ the results are:

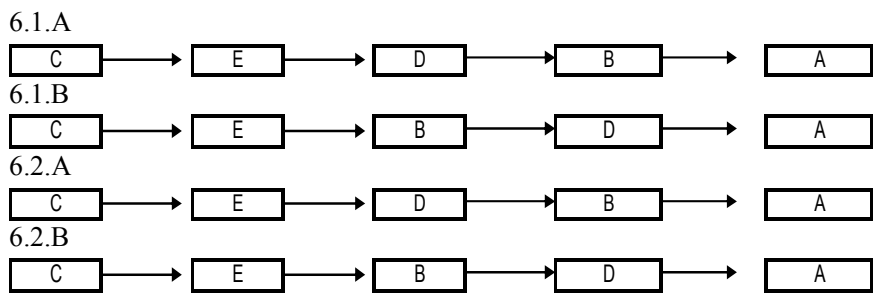

IV. CONCLUSIONS

Following the results mentioned above (Table XIII), we can obtain as conclusions that the PROMETHEE method is a very useful tool to elaborate a erosion control integral Plan. It is robust as we have confirmed changing a little the relative preference. Besides, with both methods similar results have been obtained. 
TABLE XIII

Summary Results of Promethee Methods Application to Erosion Control Plans in SAlta Province (ARgentine).

\begin{tabular}{|l|c|c|c|c|c|c|c|}
\hline Subzone & $\begin{array}{c}\text { Martin } \\
\text { Hickman }\end{array}$ & $\begin{array}{c}\text { La } \\
\text { Estrella }\end{array}$ & $\begin{array}{c}\text { Rivadavia } \\
\text { Banda Sur }\end{array}$ & Pichanal & $\begin{array}{c}\text { J. V. } \\
\text { González }\end{array}$ & $\begin{array}{c}\text { Las } \\
\text { Lajitas }\end{array}$ \\
\cline { 1 - 3 } Method & \multicolumn{7}{|c|}{ PROMETHEE, applying variable weights in each sub zone } \\
\hline 1.A & A & E & E & E & C & C \\
\hline 1.B & A & E & A & E & C & C \\
\hline 2.A & C & A & A & C & C & C \\
\hline 2.B & A & A & A & C & C & C \\
\hline \multicolumn{7}{|l}{ PROMETHEE, applying the same weights in each sub zone } \\
\hline
\end{tabular}

\begin{tabular}{|l|l|l|l|l|l|l|}
\hline $1 . \mathrm{A}$ & $\mathbf{A}$ & $\mathbf{E}$ & $\mathbf{E}$ & $\mathbf{E}$ & $\mathbf{C}$ & $\mathbf{C}$ \\
\hline $1 . \mathrm{B}$ & $\mathbf{A}$ & $\mathbf{B}$ & $\mathbf{A}$ & $\mathbf{E}$ & $\mathbf{C}$ & $\mathbf{C}$ \\
\hline $2 . \mathrm{A}$ & $\mathbf{C}$ & $\mathbf{A}$ & $\mathbf{A}$ & $\mathbf{C}$ & $\mathbf{C}$ & $\mathbf{C}$ \\
\hline $2 . \mathrm{B}$ & $\mathbf{A}$ & $\mathbf{A}$ & $\mathbf{A}$ & $\mathbf{A}$ & $\mathbf{C}$ & $\mathbf{C}$ \\
\hline
\end{tabular}

Note: 1.A and 2.A : Following the initial method of Ref. [4], 1.B and 2.B: Following method modified by the authors [6].

For this purposes, we would recommend to use Promethee II modified using the ELECTRE I weights. Besides, with usual criterion and type III pseudocriterion have been obtained the best results. We could recommend to Salta Government the following actions:

Las Lajitas: extensive farming and livestock. If it is only farming it could be with crop rotation. The livestock should be with natural forestry and foraging plants.

La Estrella: We can combine Autochthonous and high value forestry with biomass production.

Pichanal: Similar to Las Lajitas.

Martin Hickman: Autochthonous forestry, combined with some crop rotation and livestock like Las Lajitas.

Rivadavia Banda Sur: Similar to La Estrella.

Joaquin V. Gonzalez: Similar to Las Lajitas combined in some areas with high value forestry.

\section{ACKNOWLEDGMENT}

We thank to "Agencia Española para la Cooperación Internacional y el Desarrollo" (AECID) by the financing support of the project A/013294/07 titled "ELABORACION DE UN PLAN INTEGRAL DE LUCHA CONTRA LA DESERTIZACION Y LA EROSION EN EL CHACO SALTEÑO (ARGENTINA)"

\section{REFERENCES}

[1] J. M. Antón, J. B. Grau; Madrid-Valencia high-speed rail line: a route selection; ICC Transport 157, pgs, 153-161: August 2004, London.

[2] J. B. Grau, J. M. Antón, A. M. Tarquis; Election of water resources management entity using a multi-criteria decision (MCD) method in Salta province (Argentine); Proceedings WMSCI, Volume: I, pgs: 3035; 2008, Orlando, Florida, USA.

[3] J. M. Antón, Juan B. Grau, Elena Sánchez; Compromise Programming Calibration for financial analysis of firms of a common sector of business, case study for a set of Spanish banks in 1995.; Applied Financial Economics; 2006 ,U.K.

[4] J.P. Brans; Ph. Vincke, A Preferent ranking Organization Method. The PROMETHEE Method for Multiple Criteria Decision-Making, Management Science, Vol. 31, Nº 6, 647-656, June 1985.
[5] J. B. Grau, "Curso: Métodos matemáticos para la toma de decisiones", Escuela de Postgrado de la Facultad de Ciencias Naturales, Universidad Nacional de Salta, Argentina, 2003.

[6] J. B. Grau, J. M. Antón, A. M. Tarquis, D. C. Sánchez, "MCDM Methods for Waste Management Planning in a Rural Área", CITSA 2007, Vol. 134, pp. 193-209.

[7] J. M. Antón, E. Ballestero, C. Bielza. "Compromise-based approach to road project selection in Madrid metropolitan area". J. of the O. R. Society of Japan, Vol.46, No 1, 2003, pp. 99-122.

[8] J. M. Antón, J. B. Grau, D. Andina. "Electre and AHP MCDM Method and the Official choice Applied to High-Speed Railway Layout alternative election". W.S.E.A.S. Transactions on Business and Economics, Issue 1, Vol.1, Jan. 2004, pp. 64-69.

[9] J. M. Antón, J. B. Grau, E. Sánchez. "Compromise Programming in Financial Analysis for Strategic Management”. Transactions of WAC2004, Vol. 17, USA, 2004, pp. 555-560.

[10] J. M. Anton, J. B. Grau, E. Sanchez. Compromise programming calibration for financial analysis of firms of a common sector of business, case study for a set of Spanish banks in 1995. Applied financial economic, pp. 445-461, 2007.

[11]B. Roy, D. Bouyssou. Aidé Multicritère à la Décision: Méthodes et cas. Economica, Paris 1993.

[12]B. Roy. Méthodologie Multicritère d'Aide à la Décision. Economica, Paris, 1985.

[13] J.P. Brans, Ph. Vincke, B. Mareschal, "How to select and how to rank projects: The PROMETHEE method". European J. of Operational Research, vol. 44, $\mathrm{n}^{\circ} 1,1986$, pp. 138-228.

[14]J. P. Brans, B. Mareschal. "The PROMCALC and GAIA Decision Support System for Multicriteria Decision Aid", Decision Support Systems, Vol. 12, 1994, pp 297-310.

[15] T. Saaty. The Analytic Hierarchy Process, Mac Graw-Hill, New York, 1980 and 1988

[16]T. Saaty. Multicriteria Decision Making: The Analytic Hierarchy Process, AHP Series (extended edition), Vol. 1, RWS Publications, Pittsburg USA, 1996.

[17] T. Saaty. Decision Making for Leaders, AHP Series (extended edition), Vol. 2, RWS Publications, Pittsburgh USA, 1996. 